\title{
Research on Stereo Matching Algorithm based on Snake Model and Epipolar Constraint
}

\author{
Xiaotong $\mathrm{Hu}^{1, \mathrm{a}}$, Shaoying $\mathrm{Guo}^{1, \mathrm{~b}}$ \\ ${ }^{1}$ College of Computer Science and Information Engineering, Tianjin University of Science and \\ Technology, Tianjin 300222, China; \\ a1194324060@qq.com, bguosy1990@163.com
}

Keywords: Three-Dimensional Measurement, Binocular Vision, Snake Model, Epipolar Constraint

\begin{abstract}
To solve the problem that the traditional method can't perfectly achieve the feature detection of low-texture image in stereo matching, a three-dimensional measurement algorithm based on Snake Model and epipolar constraint is proposed in this paper. First, describe the edges of object image as a set of Snake points; Then search for the probably matching points on another view with the constraint of epipolar line. finally, through the reverse verification, accurate matching points will be obtained, so that the three-dimensional coordinates of matching points can be calculated according to the basic principles of binocular vision. The effectiveness and precision of this method are proved by the experiment.
\end{abstract}

\section{Introduction}

The three-dimensional measurement, which based on the stereo vision, can confirm objects' three-dimensional data exactly. It is an important research branch of image processing, and has been widely applied in industrial measurement and detection [1]. To carry out a three-dimensional measurement, a measurement platform for binocular vision is required and cameras have to be accurately calibrated [2]. Currently, many camera calibration methods can be used to confirm the relation between spatial three-dimensional coordinates and image coordinate system.

After camera calibration, which most complex and important is stereo matching. The feature extraction and matching algorithm based on grey information and image information can achieve fairly good results in common image matching. However, the methods mentioned above can't favorably meet the actual needs in this paper of finding out matching points on edge contours in the left and right images of low-texture objects. As a result, an epipolar constraint matching algorithm based on Snake Model is proposed in this paper, it can favorably match points in left and right views by searching for the intersections of Snake contour and epipolar line; finally, the three-dimensional information of objects can be obtained according to the camera calibration parameters.

\section{Stereo Matching}

Peng Hui [3] et al. proposed a matching method combining SURF operators and epipolar constraint for stereo image. As the Figure 1 shows, the method can match feature points of images taken in different perspectives.

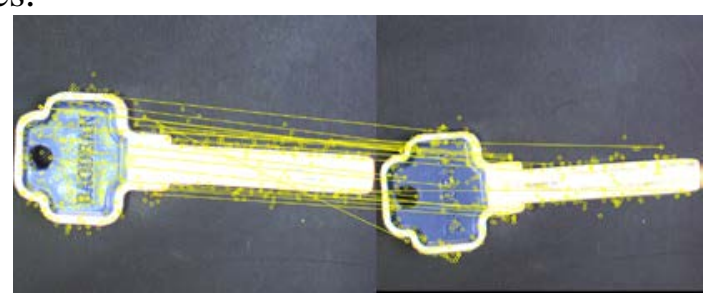

Fig.1 SURF features detect and match

It can be seen from the Figure 1 that this method can only extract a limited number of feature points for less texture information on the object and mismatching also exists. Meanwhile, the feature 
points which can be controlled in extraction are located in the edge of the object, resulting in that SURF algorithm is unable to extract edge information of objects with low-texture features.

As a result, a feature points matching with epipolar constraint based on Snake Model is proposed in this paper. Firstly, the Snake contours in left and right views will be separately extracted; later, according to epipolar constraint, the corresponding epipolar lines in the right view of the Snake contour points in the left view can be calculated; finally, the intersections of epipolar lines with the Snake contour in the right view are calculated. After the matching points with obvious errors are got rid of by reverse verification, accurate matching point pairs can be obtained.

Snake Contour Extraction. Snake Model [4] is a transformable parameter curve. It is aimed at minimizing energy, constraining its shape with internal force and guiding the behaviors by its external force, so that the parameter curve can be controlled to transform. The curve can be drawn to move to the actual contour in the entire image, which will result in an enclosed object contour with minimal energy finally. It can be known that Snake Model is a contour curve formed by the combined action of image force and external force. If $E_{s}(v)$ and $P_{s}(v)$ are respectively supposed as the image energy and external force, then the total energy $E(v)$ on contour can be defined as:

$$
\left\{\begin{array}{l}
E(v)=E_{s}(v)+P_{s}(v) \\
E_{s}(v)=\int_{0}^{1}\left(\omega_{1}(s)\left|v_{s}\right|^{2}+\omega_{2}(s)\left|v_{s s}\right|^{2}\right) \mathrm{d} s \\
P_{s}(v)=\int_{0}^{1} p(v(s)) d s
\end{array}\right.
$$

In the Equation (1), $E_{s}(v)$ defines the internal deformation energy in an extendible and flexional contour v(s), as it is controlled by two parameters: $\omega_{1}(s)$ and $\omega_{2}(s)$. These parameters control the physical behaviors and local continuity of the model. The external potential energy $P_{s}(v)$ attracts Snake contour to get close to the obvious features in the image.

According to the theory of Snake Model, an approximate initial contour has to be presupposed when the Snake active contour model is applied to search for objects. Consequently, we adopt reasonable parameters and apply the Canny operator to detect approximate edges of objects. After appropriate initialization, a series of Snake control points on the edge of objects can be obtained through continuous contour, smooth internal energy $E_{s}(v)$ and the external force $P_{s}(v)$ which controls the minimization direction of energy. Finally, the Snake contour which approaches the actual edges of objects as close as possible can be obtained by all control points are linked successively for linearization of Snake contour.

Epipolar Constraint. Epipolar constraint means a corresponding relation of one object in different images taken from different positions. By means of searching for matching points in two images, the geometrical epipolar constrained relationship can be figured out; in reverse, this constrained relationship can also applied to constrain the matching of feature points. It means to turn the hunting zone for matching feature points from a plane into an epipolar line, which not only reduces calculated quantity and saves time, but also remarkably increases matching precision.

The mathematical expression of epipolar constraint is as follows:

$$
m_{2}^{T} F m_{1}=0
$$

Therein, the $F$ refers to a fundamental matrix of $3 * 3$. It is used for making relevant description of the positional relation between two cameras. If there are two matched images, it can be calculated by several relevant matching points on the value of fundamental matrix.

Suppose $m_{1}=\left[\begin{array}{lll}u_{1} & v_{1} & 1\end{array}\right]^{T}$ and $m_{2}=\left[\begin{array}{lll}u_{2} & v_{2} & 1\end{array}\right]^{T}$ are respectively homogeneous coordinates of corresponding image points in left and right two images, then 


$$
F=\left[\begin{array}{lll}
f_{1} & f_{2} & f_{3} \\
f_{4} & f_{5} & f_{6} \\
f_{7} & f_{8} & f_{9}
\end{array}\right]
$$

Due to $F^{\prime}$ s uniqueness under the circumstance of lacking one parameter, to make the calculation simple, a non-zero parameter in $F$ can be normalized, that is, $f_{9}=1$. The number of unknown parameters in $F$ turned into 8, becoming a linear equation containing 8 unknown numbers with $m_{1}$ and $m_{2}$.

As a result, to figure out the solution of $F$ matrix, 8 pairs of matching points have to be obtained first. Once the number of matching point pairs surpasses 8 , then $F$ ' s solution can be acquired by least-square method. with the constraint matrix, the epipolar lines of every point in the other view can be drawn out.

The Epipolar Constraint Matching Method based on Snake Model. As for the Snake point set $P_{i}=\left(x_{i}, y_{i}\right) \quad(i=1,2, \ldots, n)$ of the left view figured out in the last chapter, the relation between every Snake point and its corresponding epipolar line is as follows:

$$
\left[\begin{array}{c}
x \\
y \\
1
\end{array}\right]=F_{1}\left[\begin{array}{c}
x_{i} \\
y_{i} \\
1
\end{array}\right]
$$

Therein, $F$ is the fundamental matrix between two images; $\left(x_{i}, y_{i}\right)$ refers to the points with subscript $i$ in the Snake point set of the left view. The set $(x, y)$ of $2 \mathrm{D}$ points satisfying this equation represents the points on epipolar lines in the second image. The epipolar line is expressed by the following linear equation:

$a x+b y+c=0$

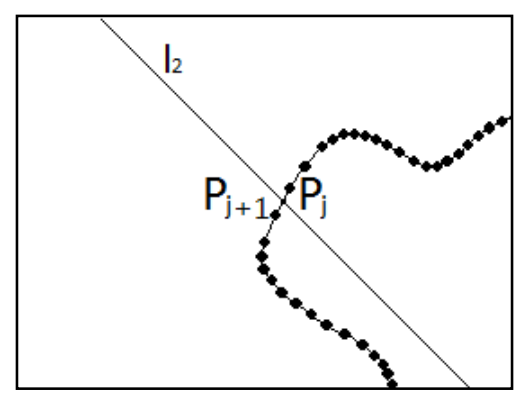

Fig.2 Sketch map of polar line and Snake contour

For the Snake point set of the right view: $P_{j}=\left(x_{j}, y_{j}\right)(\mathrm{j}=1,2 \ldots \ldots, \mathrm{s})$, as shown in the Figure 2, if there is a point $P_{j}$ making the equation (5) workable, then $P_{j}$ is assumed to be the potential matching point for $P_{i}$ in the left view; otherwise, every Snake point in the right view will be linked with consecutive points to form some corresponding segments. Two consecutive points $P_{j}$ and $P_{j+1}$, which selected from different sides of the epipolar line, will form a segment which can be expressed by following equations:

$$
\begin{cases}\frac{y-y_{j}}{y_{j+1}-y_{j}}=\frac{x-x_{j}}{x_{j+1}-x_{j}}, & y_{j+1} \neq y_{j}, \quad x_{j+1} \neq x_{j} \\ y=y_{j}, & y_{j+1}=y_{j}, x_{j+1} \neq x_{j} \\ x=x_{j}, & y_{j+1} \neq y_{j}, x_{j+1}=x_{j}\end{cases}
$$


Therein, the $\left(x_{j+1}, y_{j+1}\right)$ is the consecutive point of $\left(x_{j}, y_{j}\right)$. Then combine the equations (5) and (6) together to figure out the cross points of the epipolar line $l_{2}$ and segments $p_{j} p_{j+1}$, which may be the matching point of $P_{i}$ in the right view.

As a result, the initial matching points can be searched by several steps as follows:

Firstly, conduct traverse on Snake point set in the left image and draw its epipolar line on the right view by taking Snake points as criterion;

Secondly, take the epipolar line as a reference and conduct traverse on Snake point set in the right view to see whether there is a point intersecting with the epipolar line. If any, it is regarded as the possible matching point; otherwise, two closest Snake points above and below the epipolar line will be kept looking for in the Snake set in right view. Then the two points will be connected with each other to form a segment, as its cross point with corresponding epipolar line will be figured out to be a potential matching point.

Thirdly, repeat the above process until the traverse for the point set in the left image to be matched is finished.

After these steps, we have found out the potential matching points for all datum points in the left view, as they can be filtrated and confirmed further by reverse verification. To be specific, for several potential matching points of a certain datum point $\mathrm{P}$ in the left view, the reverse fundamental matrix F2 is taken advantage of to find out their epipolar lines and the intersection points with the contour in the left view by the same way. When an intersection point and the initial datum point $\mathrm{P}$ coincide or their Euclidean distance is less than a certain threshold value and is the least in one same group, it can be considered that this potential matching point is the corresponding matching point of datum point $P$.

In this way, the potential matching points for all datum points in the left view can be verified successively, as the points with mismatching or big errors are filtered out, excellent matching sets of two views are remained.

\section{Experimental Result}

Snake Contour Detection. During the stereo matching, the Snake points on object's edge have to be detected firstly. As shown in the Figure 3, the red curve is the Snake contour line connecting discrete Snake points. When the detected Snake contour points and generated contour line are portrayed on the original image, the expected result appears.

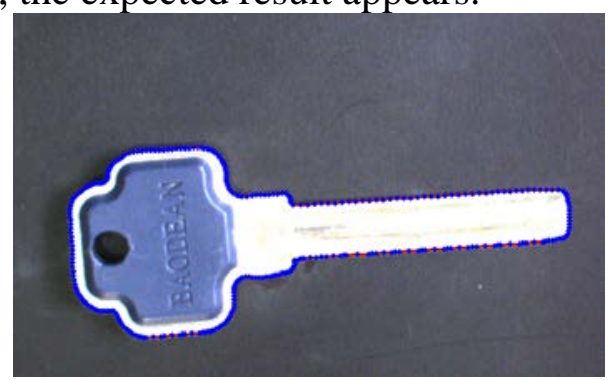

Fig.3 The Map of Snake Contour

Epipolar Constraint. Before looking up the epipolar lines, the spatial transformation relation between the left and right views has to be figured out first. Collect two target images by binocular cameras and work out the corresponding fundamental matrix $F_{1}$ and $F_{2}$ with eight-point algorithm by finding out corresponding angular points in the left and right views.

Take a plane checkerboard's image taken from different angles and draw epipolar lines of all angular points from the left view in the right view through this constraint matrix. It can be seen from the red straight line in the Figure 4 that all angular points in the right view appear on the corresponding epipolar line. 


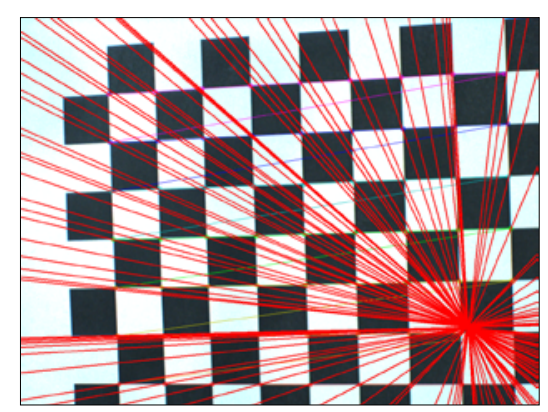

Fig.4 Epipolar constraint

Stereo Matching. For all Snake points to be matched in the left view their sole matching points in the right view can be found out through the epipolar constraint matching method based on Snake method; then the excellent matching sets between two angles can be obtained, which is shown in the Figure 5.

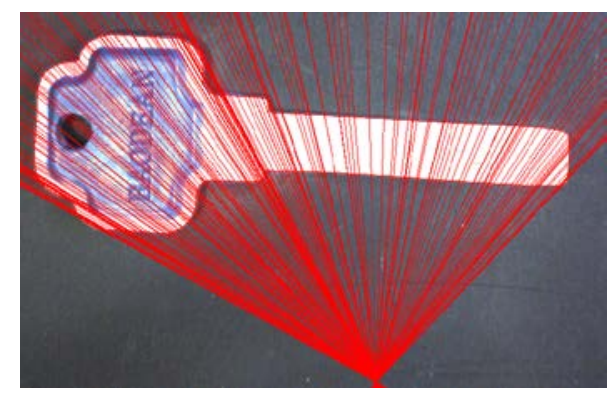

Fig.5 Stereo matching result

To observe the matching precision, 8 groups of matching data are randomly selected as shown in the Table 1. The first column represents the Snake point coordinates taken as datum points in the left view; the second shows the ultimate matching coordinates in the right view; the third indicates the corresponding coordinates of the matching points after reverse verification; the fourth shows the euclidian distance between the datum points and their reversely verified points .

Table.1 Analysis of stereo matching

\begin{tabular}{|c|c|c|c|c|c|c|}
\hline \multicolumn{2}{|c|}{ Points in the left view } & \multicolumn{2}{|c|}{ Points in the right view } & \multicolumn{2}{|c|}{$\begin{array}{l}\text { Matching points of the } \\
\text { right view }\end{array}$} & \multirow{2}{*}{$\begin{array}{c}\text { Euclidian } \\
\text { distance }\end{array}$} \\
\hline $\mathrm{X}$ & $\mathrm{y}$ & $\mathrm{x}$ & $\mathrm{y}$ & $\mathrm{x}$ & $\mathrm{y}$ & \\
\hline 466 & 496 & 634.07 & 499 & 466.029 & 496.03 & 0.042 \\
\hline 406 & 500 & 569.35 & 496 & 406.1 & 499.99 & 0.101 \\
\hline 322 & 576 & 465.27 & 562.89 & 322.03 & 576.12 & 0.124 \\
\hline 483 & 710 & 462 & 619.63 & 482.75 & 710.08 & 0.262 \\
\hline 539 & 647 & 693.49 & 674 & 539.21 & 646.98 & 0.211 \\
\hline 670 & 620 & 834.03 & 594.67 & 670.04 & 619.99 & 0.041 \\
\hline 766 & 613 & 974.1 & 602.61 & 765.99 & 613 & 0.01 \\
\hline 504 & 525 & 666 & 525.06 & 503.92 & 524.75 & 0.262 \\
\hline
\end{tabular}

Through statistical calculation on several satisfying matching points, the Euclidean distance between the datum point and reversely verified point in the left view is only 0.155 pixels, showing a fairly high matching precision.

\section{Summary}

In this paper, the actual needs of three-dimensional measurement are combined. For the stereo matching of low-texture images, the epipolar constraint matching algorithm based on Snake model is proposed. As the experiment shows, this method is simple and feasible, as it can rapidly and accurately achieve the stereo matching of objects' edge. In the future work, the three-dimensional coordinates of physical contour can be calculated with the calibrated parameters of camera, so as to analyze the shape and size of the object. 


\section{Acknowledgements}

Thanks are due to Professor Hu for assistance with the experiments and valuable knowledge which I didn’t know. I acknowledge Tianjin University of Science and Technology which provide me a good learning and research environment. And I also appreciate all the people who have contributed to this paper in some manners. Thank you!

My paper is supported by National Natural Science Foundation of China(Grant No.61502339).

Affiliations: Shaoying Guo, Tianjin University of Science and Technology, Tianjin 300222, China.

\section{References}

[1] Tang Wei, Ye Dong. 3D computer vision measurment systems [J]. Infrared and Laser Engineering, 2008 (S1) 328-332 .

[2] Zhang Zhiyong, Wang Qiong, Pu Liang,at al. Practical CCD camera calibration method [J]. Infrared and Laser Engineering. 2006 (z4) 408-413.

[3] Peng Hui, Wen Youxian, Zhai Ruifang, et al. Stereo matching for binocular citrus images using SURF operator and epipolar constraint [J]. Computer Engineering and Applications, 2011, 47 (8) 157-160.

[4] Yang Guibao, Li Ruijun, Gao Xia. An algorithm of Roof Contour Extraction Based on Snake and Dynamic Programming Optimization [J]. Journal of Inner Mongolia University (Natural Science Edition), 2015 (6) 664-671. 